Aus der chirurgischen Universitätsklinik zu Rostock (Geh. Medizinalrat Prof. W. Müiler).

\title{
Zur Statistik der operativen Dauerheilungen des Mammacarcinoms.
}

Von Dr. Hans Lindenberg, Assistenzarzt der Klinik.

Noch nicht 50 Jahre ist es her, daß man in Ärztekreisen aufgehört hat, den Krebs als eine absolut unheilbare Krankheit anzusehen. Seitdem hat sich die Chirurgie in immer steigendem Maße der Krebstherapie bemächtigt. Mit der Entdeckung der Röntgen- und Radiumstrahlen ist dem Chirurgen in neuester Zeit ein anscheinend mächtiger Bundesgenosse erwachsen; aber die Erfolge der Strahlentherapie sind noch jung und müssen erst einige Zeit überdauert haben, um Beweiskraft zu erhalten. Auch in unserer Klinik sind die Röntgenstrahlen zur Unterstützung der operativen Behandlung herangezogen worden, bei der Statistik unserer Erfolge bei Mammacarcinom habe ich aber noch nicht mit ihnen rechnen können. In den Jahren, die für mich in Betracht kamen, wurden nur ganz vereinzelte Bestrahlungen vorgenommen, noch dazu mit nach modernsten Anschauungen unzureichenden Mitteln. Darum handelt meine Statistik nur vom operativen Heilverfahren.

Die Fälle, um die es sich hier handelt, I 83 an der Zahl, sind in den Jahren I9O I bis Ende I9IO an der Rostocker chirurgischen Klinik unter Leitung von Herrn Prof. Müller operiert worden, der die Klinik im Herbst I9o I übernahm. Daher ist dieses Jahr als Ausgangspunkt gewählt worden. Die in den letzten drei Jahren operierten Fälle wurden fortgelassen, da ein Triennium als das mindeste zu gelten hat, was man an Rezidivfreiheit verlangen muß, um von Heilung sprechen zu können. Da die meisten modernen Statistiken auf drei Jahre berechnet sind, läßt sich so auch am besten ein Vergleich anstellen. Ob der Zeitraum ausreichend ist, soll an der Hand der Resultate erörtert werden. 
Die Frage nach der Entstehung des Mammacarcinoms, sowie des Carcinoms überhaupt, will ich hier nicht streifen. Dagegen möchte ich summarisch auf die Vorgeschichte unserer Kranken eingehen, ob sie uns einige Anhaltspunkte über häufigere Entstehungsursachen, vielleicht Gelegenheitsursachen des Brustkrebses gibt. Da ist zunächst die Frage der Erblichkeit. Über Carcinomerkrankungen in der Familie finde ich nur in 20 Fällen eine Angabe. Dabei ist aber in Betracht zu ziehen, daß bei der hierzulande herrschenden Krebsfurcht die Nachforschung im Interesse der Psyche des Patienten mit der äußersten Vorsicht gehandhabt werden muß. Oft wissen weder die Erkrankten, noch deren Angehörige die wahre Natur ihres Leidens. Der geringe Prozentsatz von I0,9 Carcinomfällen in der nächsten Familie kann daher keinen Anspruch auf Vollständigkeit machen. So lassen sich vielleicht auch die sehr verschiedenen Angaben in der Literatur erklären. Während Rosenstein ${ }^{1}$ ) in 3, I Proz., Gebele $e^{2}$ ) und Winiwarter ${ }^{3}$ ) in 5 Proz. ihrer Fälle erbliche Belastung angeben, fanden Horner und Poulsen ${ }^{4}$ ) in Kopenhagen 17 Proz., Wunderli5) in St. Gallen 20 Proz. Krebsfälle in der Familie. Die Statistik gibt also in dieser Frage keine eindeutige Antwort.

Leichter als nach der Erblichkeit wird es dem Arzt, die Frage nach einem Trauma zu stellen. Da tritt aber das Kausalitätsbedürfnis der meisten Menschen hindernd in den Weg, die gern für jede Erscheinung eine äußere Ursache suchen. Deshalb wird das Trauma als Ursache des Mammacarcinoms von den Beobachtern gleich niedrig gewertet; nur Horner ${ }^{6}$ ) spricht ihm größere Bedeutung für das spätere Auftreten eines malignen Tumors zu. In I I Fällen führten unsere Patienten ihr Leiden auf einen Stoß oder Schlag gegen die Brust zurück; das sind 6 Proz. Die Angaben in anderen Arbeiten schwanken zwischen 2,2 Proz.

1) Rosenstein. Arch. f. klin. Chir., Bd. 63, S. 555 .

2) Gebele. Beitr. z. klin. Chir., Bd. 29.

3) Winiwarter, Beitr. zur Statistik des Carcinoms. Stuttgart 1878.

4) Poulsen. Arch. f. Chir., Bd. 42.

5) Wunderli. Deutsche Zeitschr. f. Chir., Bd. 84.

6) Horner. Beitr. z. klin. Chir., Bd. Iz. 
$\left(\right.$ Hildebrand $\left.{ }^{1}\right)$ ) und 44 Proz. (Williams $\left.{ }^{2}\right)$ ). Es überwiegen die Zahlen um Io Proz. Williams bemerkt aber ausdrücklich, er lege dem einmaligen sozusagen akuten Trauma keinen Wert bei. Eher könne man sich schon denken, daß eine dauernd einwirkende Schädigung die Entstehung eines Carcinoms begünstige. Dieser Ansicht möchte ich mich anschließen.

Ob die Ehe von Einfluß auf die Entstehung des Carcinoms ist, läßt sich an der Hand der Zahlen ebenfalls schlecht nachweisen. Die Verheirateten überwiegen überhaupt bei Frauen in reiferem Alter, so daB es weiter nicht verwunderlich ist, wenn bei fast allen Autoren die Verheirateten sehr in der Mehrzahl sind, von 60 Proz. bei Poulsen ${ }^{3}$ ) bis 90 Proz. bei Winiwarter ${ }^{4}$ ); mit einziger Ausnahme von $\mathrm{Heath}^{5}$ ), der mehr unverheiratete $\mathrm{Pa}$ tientinnen beobachtet hat. Bei uns betrug die Zahl der Verheirateten 84,4 Proz. Wie viele von diesen Frauen gestillt haben, läßt sich leider aus den Anamnesen nicht mit Sicherheit feststellen.

Vorhergegangene Entzündung der Brustdrüse fand sich in Io Fällen $=5,4$ Proz.; dagegen haben Winiwarter 21,05 Proz., Gebele ${ }^{6}$ ) 20 Proz., Sprenge17) 30 Proz., Oldekopp ${ }^{8}$ ) 9 Proz. und Hildebrand gar 4I Proz. Man wird also auch die Mastitis als Mitursache für die Entstehung eines Carcinoms, vielleicht auf dem Narbengrund, nicht ganz vernachlässigen dürfen.

Eine wichtige Rolle in der Carcinomfrage überhaupt spielt das Alter. Das Mammacarcinom macht keine Ausnahme von der Regel, daß Krebserkrankungen vor dem 30. Jahre äußerst selten sind. Unsere jüngste Patientin war 22 Jahre alt. Ich nehme vorweg, daß unsere vier Patientinnen unter 30 Jahren die Erfahrung bestätigen, daß die Carcinome im jugendlichen Alter besonders

\footnotetext{
1) H i ldebrand. Deutsche Zeitschr. f. Chir., Bd. 25.

2) Williams. Zit. nach Schwarzkopf.

3) Poulsen. 1. c.

4) Winiwarter. 1. c.

5) He a th. Med. press. and circular 1895 . Ref. von F in sterer.

6) Gebele. 1. c.

7) Sprengel. Arch. f. Chir., Bd. 27.

8) Oldekopp. Arch. f. Chir., Bd. 24.
} 
bösartig zu sein pflegen. Drei starben schon im I. Jahr, eine im 2. Jahr nach der Operation. Dem Alter nach verteilen sich unsere Fälle folgendermaßen:

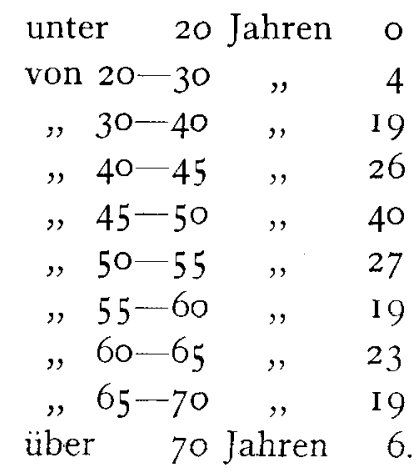

Das Jahrzehnt zwischen 40 und 50 Jahren stellt also einen weit größeren Prozentsat $z$ als die übrigen, und in diesem wiederum die zweite Hälfte. Die Menopause war bereits bei 76 Frauen eingetreten, noch nicht bei 59; in 43 Fällen fehlen die Angaben. Meiner Meinung nach kommt es wenig auf den Zeitpunkt des Aufhörens der Blutungen an, die Umwandlung des weiblichen Körpers in dieser Zeit des Übergangs erstreckt sich ja oft auf Jahre. Es ist ziemlich naheliegend, daß in einer Periode der gesamten Umwälzung schlummernde Keime einen Wachstumsimpuls erhalten und zu Neubildungen führen. Im 40.-55. Jahre sind 93 von unseren Patientinnen erkrankt; das ist etwas mehr als die Hälfte. Aber auch die späteren Jahre liefern noch einen großen Prozentsatz. Ein rasches Absinken, wie es Sprengel1) für das 7. Dezennium angibt, findet sich in unserem Material nicht. Nach diesem läßt sich also schließen: Das Mammacarcinom ist in der Hauptsache eine Krankheit der Übergangsjahre und des Alters. Darin stimmen wir mit den meisten Autoren überein, nur Sprengel und Volkmann ${ }^{2}$ ) verlegen die Hauptzeit zwischen das 50. und 6o. Jahr.

Drei Fälle von Mammacarcinom beim Manne wurden in der in Frage kommenden Zeit bei uns behandelt; einer war 50 Jahre alt, zwei in den 6oigern; ich habe sie in die allgemeine Statistik

I) Sprengel. 1. c.

2) Volkmann. Beitr. z. klin. Chir. 1875 . 
mit aufgenommen; als Einzelfälle würden sie einen statistischen Wert doch nicht besitzen.

Die Zeit, die zwischen der Entdeckung des Tumors durch die Patientinnen und der Operation verstrichen ist, läßt sich leider nur in den wenigsten Fällen mit Sicherheit feststellen. Das ist zu sehr von dem Grade der Selbstbeobachtung abhängig, bzw. von der Sorgfalt, die die einzelne ihrem Körper widmet. So wird die gebildete Frau, die von dem Vorkommen und der Bösartigkeit der Brustgeschwülste schon gehört hat, sie eher bemerken als die Arbeiterfrau. Carcinomfälle in der Familie oder der Bekanntschaft schärfen die Beobachtung. Unsere Patientinnen machten über die Zeit, seit sie den Tumor bemerkt haben, folgende Angaben:

\begin{tabular}{|c|c|c|}
\hline vor & 2 & Wochen \\
\hline seit & $2-4$ & . \\
\hline , & I -2 & Monaten \\
\hline " & $2-3$ & , \\
\hline " & $3-6$ & ", \\
\hline , & $6-12$ & $2 \quad$, \\
\hline , & $I-2$ & Jahren \\
\hline , & $2-3$ & , \\
\hline & $\begin{array}{l}3-4 \\
\text { ter }\end{array}$ & , \\
\hline
\end{tabular}

Ein gutes Drittel der Patientinnen ist also schon im I. Vierteljahr nach der Entdeckung des Tumors in die Klinik gekommen, fast $3 / 4$ wenigstens im I. Jahr. Im Durchschnitt haben also die Patientinnen die Klinik relativ früh aufgesucht.

In welchem Zustande kamen sie nun herein? Ähnlich wie Steinthal1) und andere möchte ich die Fälle rein klinisch in 4 Unterabteilungen einteilen. Zur I. Gruppe rechne ich die Geschwülste mit langsamem Wachstum, die gut verschieblich sind und noch keine Drüsenmetastasen gesetzt haben. Die 2. Gruppe umfaßt diejenigen, bei denen der Tumor schon eine Etappe weiter gerückt ist, indem er entweder die Achseldrüsen ergriffen hat, ohne daß Verwachsungen mit der Haut oder dem Muskel nachBd. 86 .

1) Ste inthal. Beitr. z. klin. Chir., Bd. 47 u. 78. Arch. f. Chir., 
Zur Statistik der operativen Danerheilungen des Mammacarcinoms. I6I

zuweisen sind oder mit der Haut verwachsen ist, während die Drüsen noch frei sind. In die dritte Gruppe rechne ich dann die mit Haut oder Muskel oder mit beiden verwachsenen Tumoren mit Drüsenmetastasen. Die 4. ist für die Fälle mit Supraclaviculardrüsen bestimmt. Wie oft in der Literatur beschrieben, stimmen auch bei uns Palpations- und pathologisch-anatomischer Befund bei den Drüsen nicht immer überein. 28 mal wurden Achseldrüsen gefühlt, die sich mikroskopisch als krebsfrei erwiesen, während 4 mal Carcinom in vorher nicht palpablen Drüsen gefunden wurde. Ich richte mich bei der Gruppeneinteilung nach dem mikroskopischen Befunde, da dieser für die Prognose ausschlaggebend ist. Unsere Fälle verteilen sich folgendermaßen:

$\begin{array}{ccc}\text { Gruppe } & \text { I : } & 35 \\ , & 2: & 8 \text { I } \\ , & 3: & 53 \\ , & 4: & \text { I } 4 .\end{array}$

Die Zeit zwischen Entdeckung des Tumors und seiner Operation verteilt sich in den einzelnen Gruppen folgendermaßen:

Gruppe I :

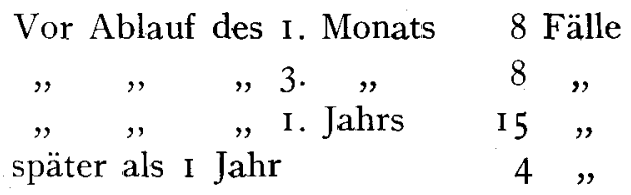

Gruppe 2:

Vor Ablauf des I. Monats I7 Fälle

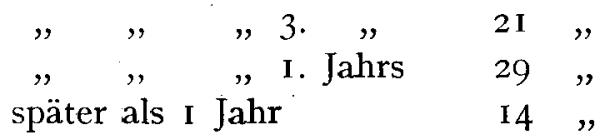

In 4 Fällen bestand der Tumor schon länger als 4 Jahre.

Gruppe 3:

$\begin{array}{ccc}\text { Vor Ablauf des I. Monats } & \text { I Fall } \\ , ", \quad \text { " 3. „ } & 5 \text { Fälle } \\ \text { " " I. Jahrs } & 23 \text { " } \\ \text { später als I Jahr } & 24 \text { " }\end{array}$

In 7 Fällen bestand der Tumor schon länger als 4 Jahre. 


\section{Gruppe 4:}

$\begin{array}{cccc}\text { Vor Ablauf des I. Monats } & \text { o Fälle } \\ ", \quad \text { " 3. " } & 4 & \text { ", } \\ \text { " " } & , \text { I. Jahrs } & 4 & , \\ \text { später als I Jahr } & 6 & \end{array}$

In 2 Fällen bestand der Tumor schon länger als 4 Jahre.

In der I. Gruppe beträgt also die Zahl der Frühfälle, d. h. derjenigen, die im I. Vierteljahr zur Operation kamen 45,7 Proz., in der 2. Gruppe 46,9 Proz, in der 3. Gruppe I I,3 Proz. und in der 4. Gruppe 28,6 Proz. Daraus geht zunächst hervor, daß die Zeit seit dem Bestehen des Carcinoms allein wenig über sein Fortgeschrittensein aussagt. Denn die Fälle der Gruppe I sind im Durchschnitt nicht älter als die der Gruppe 2, ja im Gegenteil, der Prozentsatz der verhältnismäßig früh entdeckten Fälle ist in Gruppe 2 am größten. Die I. Gruppe nimmt eine Sonderstellung ein. Die in ihr enthaltenen Fälle scheinen nur geringe Neigung zur Ausbreitung zu haben. Sie nehmen, wie wir später sehen werden, auch eine besondere Stellung in bezug auf die Dauerresultate ein. Gleichzeitige Erkrankung beider Mammae wurde 2 mal beobachtet. Beide Patientinnen sind ihrem Leiden im I. Jahre erlegen.

Vergleichen wir nun diese klinischen Befunde mit den nach der Operation, zum Teil während derselben gestellten pathologisch-histologischen Diagnosen! Die Tumoren wurden sämtlich im pathologischen Institut zu Rostock untersucht. Es wurden fast ausschließlich Carcinome beobachtet, die ihren Ursprung vom Drüsengewebe nehmen; das sind die verschiedenen Formen des Carcinoma solidum. Von dem selten beobachteten Plattenzellencarcinom (Langhans) haben wir nur einen Fall. Im einzelnen verteilen sich die Diagnosen wie folgt:

$\begin{array}{lr}\text { Carcinoma simplex } & 96 \text { Fälle } \\ \text { Scirrhus } & 40 \quad, \\ \text { Adenocarcinom } & 3 \text { I }, \\ \text { Carcinoma medullare } & 4 \quad, \\ \text { Gallertcarcinom } & 5 \text { " } \\ \text { Plattenzellencarcinom } & \text { I Fall. }\end{array}$

In 4 Fällen fanden sich Tumoren von verschiedenem Bau in 
Zur Statistik der operativen Dauerheilungen des Mammacarcinoms. I63

den einzelnen Teilen, die vielleicht als Übergangsformen anzusprechen sind. Es fanden sich:

I mal Carcinom und Scirrhus,

2 mal Scirrhus und Medullarcarcinom,

I mal Scirrhus und Adenocarcinom.

Bei einem Scirrhus ergab die Untersuchung der Achseldrüsen das Bild des Carcinoma simplex. Endlich war bei gleichzeitiger Erkrankung beider Mammae in einem Falle rechts Carcinom und links Scirrhus nachzuweisen. Das gleichzeitige Vorkommen eines gutartigen Tumors neben dem Carcinom in derselben oder der gesunden Mamma ist in meinem Material nicht zu verzeichnen. Über die Hälfte kommt bei unseren Fällen auf das einfache Carcinom, etwa $2 / 9$ auf den Scirrhus und $1 / 6$ auf das Adenocarcinom. Dies sind also die Erkrankungen, mit denen wir am meisten zu rechnen haben. Medullar- und Gallertcarcinom gehören bei uns zu den Seltenheiten. Leider finde ich in der Literatur wenig Angaben über diese Verteilung der pathologischen Befunde. Es wird meist nur über das Verhältnis der Heilungen zur Gesamtzahl der einzelnen Carcinomformen berichtet. Wunderlis ${ }^{1}$ ) Statistik stimmt ziemlich mit der meinen überein. Der Prozentsatz des Carcinoma simplex ist bei ihm etwas größer, der des Adenocarcinoms bedeutend geringer. Hildebrand ${ }^{2}$ ) findet unter 36 Fällen I I mal Scirrhus und 5 Gallertcarcinome. Das Adenocarcinom und Carcinoma simplex unterscheidet er nicht. Salomon ${ }^{3}$ ), hat I9I 3 das Material der Bierschen Klinik nach pathologisch-histologischen Gesichtspunkten bearbeitet. Er hat ähnliche Zahlen wie ich, nur für den Scirrhus viel geringere. Le e $\mathrm{ch}^{4}$ ) findet einen besonders großen Prozentsatz von Scirrhus (54 Proz.).

Das Verhältnis des klinischen zum mikroskopischen Bilde und zugleich das beider zur Dauer des Leidens möchte ich durch folgende Tabellen veranschaulichen:

I) Wunderli. 1. c.

2) $\mathrm{Hildebrand}$. 1. c.

3) Sa lomon. Arch. f. klin. Chir. IgI3.

4) L e e ch. Brit. med. journ. I9 Io, p. 73. 
I. Stadium 35 .

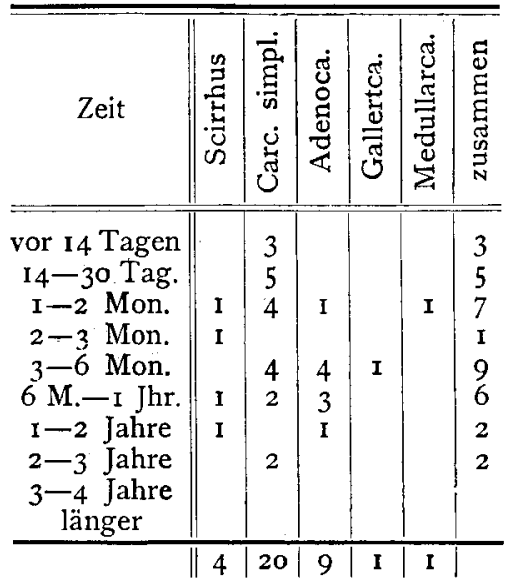

3. Stadium 53 .

\begin{tabular}{|c|c|c|c|c|c|c|}
\hline Zeit & 总 & 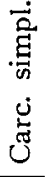 & 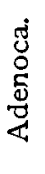 & $\frac{d}{\stackrel{d}{d}}$ & 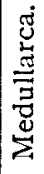 & 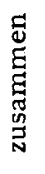 \\
\hline $\begin{array}{c}\text { vor I4 Tagen } \\
14-30 \text { Tag. } \\
1-2 \text { Mon. } \\
2-3 \text { Mon. } \\
3-6 \text { Mon. } \\
6 \mathrm{M} \text { - - I Jhr. } \\
1-2 \text { Jahre } \\
\text { 2-3 Jahre } \\
3-4 \text { Jahre } \\
\text { länger }\end{array}$ & $\begin{array}{l}\text { I } \\
\text { I } \\
4 \\
3\end{array}$ & $\begin{array}{l}3 \\
5 \\
8 \\
6 \\
2 \\
2 \\
3\end{array}$ & $\begin{array}{l}2 \\
1\end{array}$ & & & $\begin{array}{c}5 \\
0 \\
14 \\
10 \\
5 \\
2 \\
7\end{array}$ \\
\hline & I I & 30 & 9 & & & \\
\hline
\end{tabular}

2. Stadium $8 \mathrm{r}$.

\begin{tabular}{|c|c|c|c|c|c|c|}
\hline Zeit & 胥 & 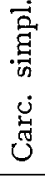 & 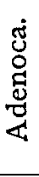 & 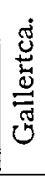 & 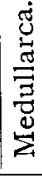 & 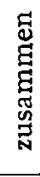 \\
\hline \multirow{10}{*}{$\begin{array}{l}\text { vor I4 Tagen } \\
\text { I4-30 Tag. } \\
\text { 1-2 Mon. } \\
2-3 \text { Mon. } \\
3-6 \text { Mon. } \\
6 \text { M.-1 Jhr. } \\
\text { I-2 Jahre } \\
\text { 2-3 Jahre } \\
\text { 3 -4 Jahre } \\
\text { Jänger }\end{array}$} & 3 & 3 & & & $I$ & 7 \\
\hline & 2 & 8 & & & & 10 \\
\hline & 5 & 4 & 2 & I & & I 2 \\
\hline & 2 & 3 & I & & I & 7 \\
\hline & 3 & I & 4 & I & I & IO \\
\hline & 6 & I I & 2 & & & 19 \\
\hline & & 4 & 2 & & & \\
\hline & & I & & & & \\
\hline & I & I & & & & \\
\hline & & 3 & & I & & \\
\hline & 22 & 39 & & 3 & 3 & \\
\hline
\end{tabular}

4. Stadium I4.

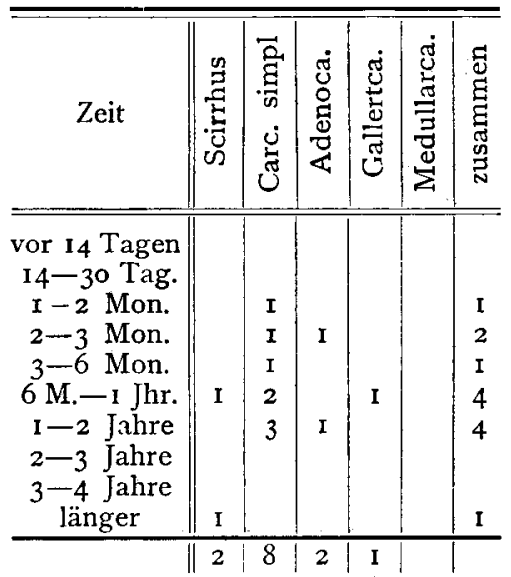

Aus diesen Tabellen geht hervor, daß dem I. klinischen Stadium angehören :

29 Proz. der Fälle von Adenocarcinom,

\begin{tabular}{|c|c|c|c|c|c|}
\hline & " & ", & $"$ & , & Carcinoma simplex \\
\hline & $"$ & "' & , & " & Scirrhus, \\
\hline & & & & , & Medullarcarcinom, \\
\hline & & , & , & , & Gallertcarcinom. \\
\hline
\end{tabular}

Die letzten Zahlen sind eine gute Illustration für die Mißlichkeit der Anwendung der prozentualen Berechnung bei kleinen 
Zur Statistik der operativen Dauerheilungen des Mammacarcinoms. I 65

Zahlen. Da von 5 Gallertcarcinomen und 4 Medullarcarcinomen je eins in einem klinisch noch nicht fortgeschrittenen Zustand zu uns kam, erhalte ich die Zahlen, 20 Proz. bzw. 25 Proz., die ohne Berücksichtigung der absoluten Menge zu falschen Schlüssen führen könnten. Auf die Prozentstatistik bei kleinem Material werden wir bei der Besprechung der Resultate zurückkommen müssen. Das Adenocarcinom scheint also verhältnismäßig am wenigsten Neigung zum schnellen Ausbreiten zu haben. Halstedt $^{1}$ ) und Salomon ${ }^{2}$ ) heben dies ebenfalls hervor. Dies entspricht insofern seinem anatomischen Charakter, als es dem normalen Bau des Ausgangspunktes, den Drüsenacinis, am nächsten steht. Den kleinsten Prozentsatz für das I. Stadium stellt der Scirrhus, von dem 55 Proz. dem 2., 27,5 Proz. dem 3. und 7,5 Proz. dem 4. Stadium angehören. Das Carcinoma simplex stellt 40,5 Proz. beim 2., 3I Proz. beim 3. und 7,5 Proz. beim 4. Stadium. Nach ihrer Neigung zum Weiterschreiten lassen sich die hauptsächlichsten Carcinomformen in folgender Reihenfolge anordnen:
I. Adenocarcinom,
2. Carcinoma simplex,
3. Scirrhus.

Im 2. Stadium verschiebt es sich insofern, als der Scirrhus an I. Stelle, das Carcinoma simplex an 2. Stelle und das Adenocarcinom an 3. Stelle rangiert. Im ganzen macht es den Eindruck, als ob es in jeder Krebsform langsam und schnell wachsende Fälle gibt. Besonders scheint dies beim Scirrhus zuzutreffen, den man nicht schlechthin als den langsam wachsenden Krebs bezeichnen kann.

Der Sitz des Tumors war $98 \mathrm{mal}$ die linke, $83 \mathrm{mal}$ die rechte Mamma und 2 mal waren beide Mammae ergriffen. Im einzelnen war die Lage der Tumoren folgendermaßen:

\begin{tabular}{lr}
\multicolumn{2}{c}{ Rechts } \\
Oberer äuBerer Quadrant & $25 \mathrm{mal}$ \\
Oberer innerer Quadrant & 9, \\
Unterer äußerer Quadrant & 10, \\
Unterer innerer Quadrant & 2, \\
Obere Hälfte & $8 "$, \\
Untere Hälfte & 2,
\end{tabular}

I) $\mathrm{H}$ a ls tedt. Annals of surgery 1907, Bd. 46.

2) Salomon. 1. c. 


\begin{tabular}{|c|c|c|}
\hline ÄuBere Hälfte & 4 & $\mathrm{ma}$ \\
\hline Innere Hälfte & I & , \\
\hline Zentral & I 3 & , \\
\hline Ganze Mamma & I I & , \\
\hline \multicolumn{3}{|l|}{ Links } \\
\hline Oberer äußerer Quadrant & 40 & " \\
\hline Oberer innerer Quadrant & II & \\
\hline Unterer äuBerer Quadrant & 5 & " \\
\hline Unterer innerer Quadrant & I & \\
\hline Obere Hälfte & I I & , \\
\hline Untere Hälfte & I & , \\
\hline Äußere Hälfte & 5 & " \\
\hline Innere Hälfte & 2 & , \\
\hline Zentral & I 6 & \\
\hline Ganze Mamma & 8 & \\
\hline
\end{tabular}

Die linke Mamma ist also etwas stärker beteiligt als die rechte, doch nicht in einem Maße, daß man daraus besondere Schlüsse ziehen könnte. Davor warnt schon Win iwarter ${ }^{\mathbf{1}}$ ) bei einer Kritik der Statistik von Henning ${ }^{2}$ ), der ein Überwiegen der rechtsseitigen Erkrankungen findet und dies mit größeren Schädigungen der rechten Brustdrüse in Verbindung bringt. Winiwarter und die übrigen Autoren beobachteten übereinstimmend ein Vorwiegen der beiden oberen äußeren Quadranten und überhaupt der oberen und auch der äußeren Hälfte. Der untere innere Quadrant ist am wenigsten beteiligt.

Bei der operativen Behandlung des Mammacarcinoms kann man erst wirklich von Erfolgen sprechen, seit Moor e ${ }^{3}$ ) I 867 gelehrt hat, die ganze Brustdrüse als erkrankt anzusehen, und nicht nur den Tumor, sondern die ganze Mamma zu entfernen. Mit der fortschreitenden Kenntnis von den Wegen, auf denen das Mammacarcinom sich verbreitet, die besonders von Volkmann ${ }^{4}$ ) Küster ${ }^{5}$ ), Heidenhein ${ }^{6}$ ), Gerota ${ }^{7}$ ), Stiles ${ }^{8}$, GroB-

I) Winiwarter. 1. c.

2) H e nning. Arch. f. Gyn., Bd. 2, S. 38 I.

3) Moore. Zit. nach Winiwarter.

4) Volkmann. I. C.

5) $\mathrm{K} \ddot{\mathrm{u}} \mathrm{s}$ t e r. Chirurgenkongre $\mathrm{B}$ I 883 .

6) $\mathrm{H}$ eide $n$ h ein. Arch. f. Chir. 1889, Bd. 39.

7) Ge rota. Arch. f. klin. Chir., Bd. 29.

8) Stiles. Edinburg Journal 1892. 
$\operatorname{mann} 1$ ) und Rotter ${ }^{2}$ ) gefördert wurde, wurden die Operationsmethoden immer radikaler. Inzwischen hatte $\mathrm{K}$ üster I 883 , wie für alle Carcinome, auch für die der Mamma, die Ausräumung der regionären Lymphdrüsen gefordert. R otter wies nach, daß ohne Infektion des Pectoralis maior und seiner Fascie, bei verschieblichem Tumor, sich infizierte Lymphdrüsen an der Rückseite des Pectoralis maior fänden. Er wies in I I von 35 untersuchten Fällen nach, daß Lymphgefäße der Mamma den Pecto. ralis maior durchbohrten und so die Keime frühzeitig in die retromuskulären Drüsen brächten. GroBmann hatte dies unter 30 Fällen nur 3 mal nachweisen können. R ot te ${ }^{3}$ ) forderte daher generell die Entfernung der Sternalportion des Pectoralis maior sowie des ganzen Pectoralis minor; letzterer störe den Zugang zur Achselhöhle und sei oft selbst infiziert. Darüber hinaus räumt Halstedt4) in jedem Falle auch die Supraclaviculardrüsen aus und reseziert $\mathrm{zu}$ diesem $Z$ wecke temporär die Clavicula.

Unsere Therapie dem Mammacarcinom gegenüber ist in der in Frage kommenden Zeit die möglichst radikale Operation gewesen und wird es voraussichtlich noch lange bleiben. In den letzten Jahren wurden nach der Entfernung des Tumors Röntgenbestrahlungen ausgeführt, doch sind unsere Erfahrungen noch nicht groß genug, um mit Sicherheit einen Einfluß der Strahlen erkennen zu lassen. Die Indikation zur Operation wurde ziemlich weit gefaßt und als inoperabel nur solche Fälle abgelehnt, die nachweisbar innere Metastasen hatten, sowie einige Fälle von Cancer en cuirasse. Wieviel es im ganzen waren, läßt sich nicht genau ermitteln, da die Abweisung dann meistens schon in der Poliklinik bzw. in der Privatsprechstunde erfolgt ist. Weder Ulceration, noch fühlbare Supraclaviculardrüsen wurden als Kontraindikation angesehen. Küttners ${ }^{5}$ ) Ansicht, daß sich in jedem Falle, in dem Supraclaviculardrüsen gefunden werden, auch schon endothorakale, Drüsenmetastasen anzu-

I) Gro Bmann. Gekrönte Preisschr. 1896.

2) Rotter. Arch. f. Chir., Bd. 58, S. 346 .

3) Rot ter. Berliner klin. Wochenschr. I896, S. 69.

4) $\mathrm{Halstedt}$. Annals of surgery I907, Bd. 46.

5) Küt tn e r. Beitr. z. klin. Chir., Bd. 36 . 
nehmen seien, ist durch Finstere ${ }^{1}$ ) an der Hand von drei gegenteiligen Obduktionsbefunden widerlegt worden. Außerdem teilt Finsterer ${ }^{1}$ ) noch drei über 4 Jahre rezidivfrei gebliebene Fälle mit, das gleiche tun Gulecke2) und Pilcher ${ }^{3}$ ); im großen und ganzen sind allerdings diese fortgeschrittenen Carcinome nicht mehr zu heilen. - Hohes Alter wurde an sich nicht als eine Kontraindikation betrachtet, waren doch 25 unserer Patientinnen über 65, 6 über 7o Jahre alt; wohl aber hat fortgeschrittene Kachexie öfters einschränkend auf unser Handeln gewirkt und Veranlassung gegeben, die Ausdehnung der Operation zu beschränken. So sind in $2 \mathrm{I}$ Fällen Supraclaviculardrüsen gefühlt, aber nur in I 4 Fällen ausgeräumt worden. Die prinzipielle Ausräumung der Supraclaviculardrüsen nach $\mathrm{Hal}$ stedt ist bei uns nicht durchgeführt worden. Hier muß man Küttner ${ }^{4}$ ) zustimmen, daß die Supraclaviculardrüsen nur eine der Etappen sind, in denen das Carcinom sich weiter verbreitet, daß also die Größe des Eingriffs nur ganz selten die Weiterentwicklung sicher aufhält, dagegen die Gefahren des Eingriffs sehr vermehrt. Bei uns werden die Supraclaviculardrüsen also nur ausgeräumt, wenn verdächtige palpabel sind und der Allgemeinzustand einigen Erfolg verspricht. Seit Mitte 1904 wurde an der Rostocker Klinik die Operation nach Rotters Vorgehen ausgeführt: Wegnahme der erkrankten Mamma unter geringer Schonung der Haut, im Zusammenhang mit dem Inhalt der Achselhöhlen und beiden Brustmuskeln; häufig wurden auf einen durch Zusammenziehen nicht zu deckenden Defekt Thierschsche Läppchen transplantiert. In dieser Art sind I20 Fälle operiert worden. Aber auch schon vorher wurde der ganze Pectoralis maior oder wenigstens eine Schicht desselben entfernt. In fünf Fällen wurde nur die Fascie weggenommen.

Während der größte. Teil der modernen Autoren einer möglichst ausgedehnten Operation das Wort redet, mahnen andere zur vorsichtigen Auswahl. Greenough ${ }^{5}$ ) sagt geradezu:

I) Finsterer. Deutsche Zeitschr. f. Chir. 1907, Bd. 89, S. 143.

2) Gulecke. Arch. f. Chir., Bd. 64 .

3) Pilcher. Annals of surgery 1907, Bd. 46, S. 67.

4) Küttner. I. $c$.

5) Greenough. Annals of surgery 1907. 
Zur Statistik der operativen Dauerheilungen des Mammacarcinoms. I69

Inkomplette Operationen in leichten Fällen geben bei weitem bessere Resultate als ausgedehnte. Ihm scheint die Statistik von $\mathrm{Heurtaux}$ ) in Nantes recht zu geben, der nur die Brustdrüse wegnimmt und die Achselhöhle ausräumt und von dessen 284 Operierten nach 4 Jahren noch I 23 am Leben waren; das sind 43,3 Proz.! Warren hat 2 Serien von 50 Patientinnen operiert; die I. Serie nach Volkmann unter Wegnahme nur der Pectoralisfascie; die 2. nach Rotter. Er erzielte in der I. Serie I4, in der 2. Serie I2 Heilungen!

Was ist nun aus unseren 183 Kranken geworden?

Der Operation als solcher, das heißt der Größe des Eingriffs, ist keine Patientin erlegen. 13 sind jedoch im Anschluß an die Operation gestorben. Das sind im ganzen 7, I Proz. Der Prozentsatz ist sehr groß, größer als er in modernen Statistiken zu sein pflegt. So geben Dollinger ${ }^{2}$ ) I,5 Proz., Mauclaire ${ }^{3}$ ) 2 Proz., Wunderlit) 2,5 Proz., P. Schmidt 5 ) 3 Proz., Finsterer ${ }^{6}$ ) 5,2 Proz., H. Schmidt 7 ) 5,2 Proz., Schwarz ${ }^{8}$ ) 6,9 Proz., Dietrich ${ }^{9}$ ) 7,6 Proz., Salomon ${ }^{10}$ ) 0,92 Proz. an.

Es hängt vielleicht damit zusammen, daß in der Rostocker Klinik die Operation auch in sehr fortgeschrittenen Fällen unbekümmert um Statistik noch versucht wird. Unter den Verstorbenen befindet sich eine Patientin mit Diabetes und zwei mit schwerem Herzfehler. Bei einem Falle handelt es sich um ein beiderseitiges Carcinom, wobei auch die linke Supraclaviculargrube mit ausgeräumt wurde. Ein Fall von Sepsis ging von einem Decubitus am Kreuzbein aus, während die Operationswunde primär heilte. Die Todesfälle verteilen sich folgendermaßen:

I) $\mathrm{H}$ e urta ux. Zit. auf dem Congr. intern. de chir., Brüssel 1908.

2) Dollinger. Congr. intern. de chir., Brüssel 1908.

3) Ma uclaire. Gazette des hôpitaux I9Io, Nr. 8I.

4) Wunderli. l. c.

5) P. Schmidt. Deutsche med. Wochenschr. 1904.

6) Finsterer. 1. c.

7) H. Schmidt. Deutsche Zeitschr. f. Chir. I887, Bd. 26.

8) Schwarz. Beitr. z. klin. Chir., Bd. 46.

9) Dietrich. Deutsche Zeitschr. f. Chir, 1892, Bd. 33.

Io) Salomon. I. c. 


$\begin{array}{ll}\text { Erisypel bzw. Sepsis } & 4 \\ \text { Embolie } & 3 \\ \text { Pneumonie } & \text { I } \\ \text { Apoplexie } & \text { I } \\ \text { Diabetes (Coma) } & \text { I } \\ \text { Endocarditis } & \text { I } \\ \text { Herzschwäche } & \text { I } \\ \text { Myodegeneratio cordis } & 2\end{array}$

Von diesen Todesfällen waren allein 4 im Jahre I902 und 5 im Jahre 1907, während sich die übrigen 4 auf 7 Jahre verteilen. Es soll dies nur ein Hinweis sein, wie der Zufall manchmal Statistiken beeinflussen kann, wenn es sich um absolut kleine Zahlen handelt.

Über die Schicksale der übrigen Kranken habe ich mich auf folgende Weise unterrichtet. Einen kleinen Teil, der in Rostock wohnte, bzw. die Klinik wieder aufsuchte, habe ich persönlich nachuntersuchen können. Ferner habe ich Fragebogen an die Kollegen gesandt, die uns die Frauen zur Operation geschickt hatten. Hierauf habe ich viele zum Teil sehr ausführliche Antworten bekommen, wofür ich den Kollegen an dieser Stelle meinen herzlichsten Dank sage, ebenso den Gemeindeschwestern und Ortsvorstehern, die mich bereitwilligst unterstützt haben, wo ärztliche Auskunft nicht zu erlangen war. So konnte ich über I 76 Fälle zuverlässige Nachricht erhalten; meistens nicht nur über erfolgten Tod, sondern auch über dessen Ursache und das Befinden vorher. Von 7 Patientinnen konnte ich nichts mehr erfahren, teils weil ihre Personalien ungenau waren, teils weil sie aus Mecklenburg unbekannt ins "Ausland“ verzogen sind.

Die Hauptfrage bei der Verwertung des Materials ist natürlich: wann kann man ein Carcinom der Mamma als geheilt betrachten? oder wann kann man wenigstens die begründete Erwartung hegen, daß das Leiden endgültig beseitigt ist? Volkman $n^{1}$ ) hat bekanntlich ausgesprochen: ist nach der Operation I Jahr verstrichen, so darf man hoffen, nach 2 Jahren aber mit einer gewissen Wahrscheinlichkeit erwarten, daß kein Rezidiv

I) Volkmann. 1. c. 
Zur Statistik der operativen Dauerheilungen des Mammacarcinoms. I 7 I

mehr eintritt. Nun lehrt die Erfahrung tatsächlich, daß der weitaus größte Teil der Rezidive wie der Metastasen sich im Laufe des I. Jahres zeigt, ein bedeutend kleinerer im 2., aber auch die im 3. Jahre auftretenden Rückfälle beider Art lassen sich noch nach Prozenten berechnen, und so haben schon in den 8 o er Jahren des vorigen Jahrhunderts die meisten Autoren 3 Jahre als die Mindestzeit festgesetzt, die verstrichen sein muß, bevor man von „Krebsheilung“" sprechen kann. In den letzten beiden Jahrzehnten wurde mehrfach die Forderung aufgestellt, erst nach 5 Jahren Rezidivfreiheit die Patientin als geheilt anzusehen, so von Steinthal ${ }^{1}$ ) und Greenough ${ }^{2}$ ). Mauclaire ${ }^{3}$ ) behauptet sogar, daß erst nach 3 Jahren die meisten Rezidive aufträten, eine Beobachtung, die sonst noch nirgends gemacht worden ist. Steiner ${ }^{4}$ ) dagegen gibt an, daß bei seinem Material der Unterschied sehr gering sei, ob nach 3 oder nach 5 Jahren die Grenze gezogen werde. Ich habe unsere Resultate deshalb auf 3 und auf 5 Jahre völliger Gesundheit abgestimmt, um zur Klärung dieser Frage beizutragen. Daß auch die Fünfjahrgrenze keine absolut sichere ist, beweisen die vielen bekannten Fälle von Spätrezidiven. Wir haben nach 5 Jahren noch 4 Fälle von Spätrezidiven bzw. Metastasen beobachtet, wovon eines nach 7 , eines nach 9 Jahren auftrat, beide in der Wirbelsäule. Förster ${ }^{5}$ ) hat je einen Fall nach IO, II, 13, I6, 20 Jahren; Verneui16) sogar einen nach 33 Jahren. Labhardt 7 ) hat dieses Kapitel ausführlich behandelt. Meistens handelt es sich bei den Spätfällen um Carcinomerkrankungen anderer Organe, und es läßt sich ohne Obduktion und genaue histologische Untersuchung nicht feststellen, ob es sich um eine Metastase des Tumors oder um eine neue Krebserkrankung handelt. Interessant waren in diesem Sinne zwei unserer Fälle.

I. Frau O. wurde im Jahre Igor wegen eines Carcinoms der

I) Steint hal. 1. c.

2) Greenough. 1. c.

3) $\mathrm{Mauclaire.} \mathrm{1.} \mathrm{c.}$

4) Steiner. Deutsche Zeitschr. f. Chir., Bd. 82.

5) Förster. Zit. nach $L a b h a r d t$.

6) Verneuil. Zit. nach $L a b h a r d t$.

7) Labhardt, Beitr. z. klin. Chir., Bd. 33. 
rechten Mamma operiert. Im Jahre 1913 kam sie wieder mit einem Cöcaltumor, der sich histologisch als Spindelzellensarkom erwies.

2. Bei Frau D. wurde I 902 ein Carcinoma simplex entfernt. I 9 I I trat an der Cervix uteri ein Plattenepithelkrebs auf.

Hier handelte es sich um histologisch anders geartete bösartige Geschwülste, also sicher nicht um Metastasen. Beiläufig möchte ich hier bemerken, daß wir mehrfach Fälle gehabt haben, bei denen früher auswärts ein gutartiger Tumor aus einer der beider Mammae entfernt worden ist.

Von unseren Operierten bekamen 77 ihr Rezidiv bereits. im I. Jahr. Das sind 42, I Proz. Die Zahl erscheint aber erst in ihrer ganzen Bedeutung, wenn man von der absoluten Menge diejenigen abzieht, die kein Rezidiv bekommen haben; das sind die an den Folgen der Operation oder an interkurenter Krankheit Gestorbenen, sowie die dauernd Geheilten, und diejenigen, von deren Schicksal wir nichts in Erfahrung gebracht haben. Es gingen zugrunde oder leben noch mit inoperablem Rezidiv Io8. Von diesen Rezidiven traten 77 schon im I. Jahre auf; das sind 7 I,2 Proz.

\begin{tabular}{|c|c|c|c|c|c|}
\hline 12 & $\mathrm{im}$ & 2. & Jahre & I I I & Proz. \\
\hline 9 & ", & 3. & ," & 8,3 & , \\
\hline 4 & ," & 4. & ," & 3,7 & , \\
\hline 2 & ", & 5. & ", & $\mathbf{I}, 8$ & , \\
\hline I & ', & 6. & ", & 0,9 & , \\
\hline I & $"$ & 7. & ", & 0,9 & , \\
\hline 1 & ", & 8. & ", & 0,9 & , \\
\hline O & , & 9. & ", & O & , \\
\hline I & , & IO. & , & 0,9 & , \\
\hline
\end{tabular}

Fast $3 / 4$ aller Rezidive traten also im I. Jahre auf, über $4 / 5$ in den ersten zwei Jahren. Volkmann hat doch nicht so ganz unrecht gehabt! Jedes weitere Jahr gibt allerdings eine größere Sicherheit; wir sehen das 3. Jahr noch etwa $3 / 4$ so stark beteiligt wie das 2. Im 4. Jahr gibt es nur noch knapp halb so viel Wiedererkrankungen wie im 3., im 5. wieder um die Hälfte weniger als im 4. Jahr. Von Io8 Rezidiven traten 6 zwischen dem 3. und 5. Jahre auf. Später kommen die Fälle nur noch vereinzelt vor. 
Nunmehr muß ich auf die Einzelheiten meiner Statistik näher eingehen. Ich will die Heilungen berechnen nach dem Prinzip der dreijährigen und der fünfjährigen Periode. Für erstere kommen I83, für letztere I 53 Fälle in Betracht. Man kann sie nun in zweierlei Form zur Darstellung bringen.

I. die nach 3 bzw. 5 Jahren noch Lebenden,

2. die nach 3 bzw. 5 Jahren noch Rezidivfreien.

Wichtig für die Statistik ist auch, wie man mit den an interkurrenter Krankheit Verstorbenen verfährt. Man kann sie nicht zu den Rezidiven rechnen, darf sie auch nicht für die Dauerheilungen verwerten, wenn sie nicht mindestens den in Frage stehenden Zeitraum, also 3 bzw. 5 Jahre, rezidivfrei überlebt haben und eine andere Todesursache ärztlich festgestellt ist. Legen wir also zunächst eine 3 jährige Periode zugrunde, so erhalten wir folgende Zahlen:

\begin{tabular}{lc} 
Von & I 83 Fällen \\
\hline haben wir keine Antwort über & 7 \\
starben an den Folgen der Operation & I3 \\
starben an interkurrenter Krankheit & 5 \\
starben an Rez. bzw. Met. im I. Jahr & 54 \\
im 2. Jahr & I 5 \\
im 3. Jahr & I 2 \\
\hline Mithin leben nach 3 Jahren & $77=42$, I Proz. \\
Davon haben aber schon ein Rezidiv & 17 \\
\hline Mithin leben nach 3 Jahren rez.-frei & $60=32,7$ Proz.
\end{tabular}

Für die fünfjährige Periode kommen nur die bis I 908 einschließlich operierten Fälle in Betracht, das sind davon blieben ohne Antwort $\frac{\mathrm{I} 53}{7}$ starben an den Folgen der Operation 12 starben an interkurrenter Krankheit 8 starben am Rez, in den ersten 3 Jahren 67

$$
\begin{array}{ll}
\text { im 4. Jahr } & 6 \\
\text { im 5. Jahr } & 5
\end{array}
$$

$\frac{\text { im 5. Jahr }}{\text { Mithin leben nach } 5 \text { Jahren }} \quad \frac{5}{48=3 \mathrm{I}, 4 \text { Proz. }}$

Davon haben bereits ein Rezidiv 5

Mithin leben rez.-frei nach 5 Jahren $43=28$ Proz.


Von den 43 geheilten Patientinnen hatten 3 bald nach der Operation ein Lokalrezidiv, welches vor länger als 5 Jahren operiert wurde.

Die Statistik sagt also, wenn man die nackten Zahlen reden läßt: Der Unterschied zwischen den „Heilungen“ nach 3 oder 5 Jahren beträgt 5 Proz.! Hierbei muß man aber berücksichtigen, daß die fünfjährige Statistik I 2 von I3 Todesfällen mit übernehmen muß, daß alle nicht eingegangenen Antworten und eine erheblich größere Zahl an interkurrenter Krankheit Gestorbener ihr Konto belastet. Es hieße neue Fehlerquellen eröffnen, wollte ich den Versuch machen, diese größere Schädigung zahlenmäßig festzustellen. Aber das möchte ich doch behaupten, daß der wirkliche Unterschied nur höchstens 3 Proz. beträgt, es mithin im großen und ganzen von geringer Wichtigkeit ist, ob man die Heilungsgrenze auf 3 oder 5 Jahre festsetzt. Ganz sicher vor Rezidiv sind alle unsere Patientinnen nicht; auch von denen, die 5 Jahre rezidivfrei überlebten, sind noch 3 später gestorben, eine lebt noch mit Metastase. Das sind immerhin noch 9,3 Proz. So müssen wir denn Volkmanns Ausspruch dahin modifizieren: Nach 2 Jahren kann man hoffen, nach 3 Jahren wird die Hoffnung verstärkt, nach 5 Jahren ist die Wahrscheinlichkeit der Wiedererkrankung sehr gering.

Betrachten wir nunmehr die geheilten Fälle näher! Da taucht zunächst die Frage auf: sind es etwa besonders geartete Fälle, bei denen wir erfolgreich operieren, sei es, daß sie noch nicht so weit fortgeschritten sind, sei es, daß sie einer minder bösartigen Geschwulstart angehören. Daß „Krebs“ und „Krebs“ ein Unterschied ist, ist ja eine alte Wahrheit; und bei dem Vergleich zwischen klinischem Stadium und histologischer Diagnose hat sie sich uns wieder aufgedrängt. Von den Patientinnen, die jetzt noch rezidivfrei leben, denen ich noch diejenigen zuzähle, die an interkurrenter Krankheit nach 3 rezidivfreien Jahren gestorben sind, also von 50 , gehören

dem I. klinischen Stadium an: (beweglicher Tumor ohne Drüsenmetastasen)

dem 2. klinischen Stadium (Achseldrüsen, Tumor beweglich, oder keine Achseldrüsen, aber Tumor verwachsen): 
Zur Statistik der operativen Dauerheilungen des Mammacarcinoms. I75

dem 3. klinischen Stadium (Achseldrüsen und Tumor verwachsen):

dem 4. klinischen Stadium (Supraclaviculardrüsen): $\mathrm{O}$

Diese Zahlen gewinnen aber erst ihre volle Bedeutung, wenn man sie den absoluten Zahlen ihrer Gruppen gegenüberstellt.

I. Stadium: 35 Fälle: 24 Heilungen $=68,5$ Proz.

2. $\quad: 8 \mathrm{I} \quad,: 22 \quad, \quad=27, \mathrm{I} \quad$,

3. " : 53, : $4 \quad, \quad=7,6$ "

4. $, \quad:$ I $4, \quad$ : $0 \quad, \quad=0$,

Bekommen wir also die Fälle im Beginn ihrer klinischen Entwicklung, die nicht immer mit der Zeit des Bestehens des Leidens übereinzustimmen braucht, so haben wir $2 / 3$ Wahrscheinlichkeit, heilen zu können. Hat der Tumor aber schon die I. Etappe seines Zuges durch den Körper erreicht, so sinken die Aussichten unter $1 / 3$, hat er noch weiter um sich gegriffen, auf $1 / 12$, um bei $\mathrm{Er}$ krankung der Supraclaviculardrüsen $=\mathrm{o}$ zu werden. Uns ist kein Fall mit Supraclaviculardrüsen länger als $2^{1 / 2}$ Jahre rezidivfrei geblieben; die oben erwähnten Heilungen von Finsterer, Gulecke und Pilcher werden uns aber doch immer wieder ermutigen, die gefühlten Supraclaviculardrüsen mit fortzunehmen, denn eine Lebensverlängerung ist doch vielleicht noch zu erreichen.

Die histologische Diagnose bei unseren geheilten Fällen war:

Adenocarcinom

Scirrhus

Carcinoma spl.

Medullarcarcinom

Gallertcarcinom Io Heilungen von 3 I Fällen $=32,3$ Proz.

Die letzten beiden Prozentsätze haben natürlich geringen Wert.

Das Adenocarcinom nahm schon unter den Fällen des I. klinischen Stadiums die erste Stelle ein, interessant ist aber der Vergleich zwischen dem Carcinoma simplex und dem Scirrhus. Während 2I Proz. des einfachen Carcinoms dem I. Stadium angehören und nur to Proz. der Scirrhen, die ersteren also eigentlich bedeutend günstigere Chancen zu bieten scheinen, sehen wir den Unterschied bei den Heilungen fast ausgeglichen. Vermutlich hängt es damit zusammen, daß der Scirrhus zwar oft rasch auf die Drüsen übergreift, aber im ganzen ein langsames Wachstum 
zeigt. Vergleichen wir hiermit die Fälle einiger anderer Autoren: Halstedt ${ }^{1}$ ) findet ebenfalls die günstigste Heilungsziffer für das Adenocarcinom, bei dem er 75 Proz. Dauererfolge hat, für das Carcinoma medullare 48 Proz., für zirkumskripten Scirrhus 46 Proz. und für infiltrativen 22 Proz. Warren ${ }^{2}$ ) hatte beim Adenocarcinom 662/3 Proz. Heilungen - aber nur 3 Fälle. Von 26 einfachen Carcinomen wurden 6 geheilt, das sind 23 Proz., beim Scirrhus hatte er 43 Proz. Heilungen. Er ist der einzige, der eine größere Zahl von Medullarcarcinomen hat, nämlich 40 mit nur 3 Dauerheilungen, das sind 7,5 Proz.! Die Verteilung der Heilungsquoten bei Salomons ${ }^{3}$ ) Material ist hier nicht zum Vergleich heranzuziehen, da er nach ganz anderen Gesichtspunkten einteilt. Aber auch er findet für die Adenocarcinome eine besonders günstige Prognose. Leider ist nur in sehr wenigen Arbeiten das Verhältnis zwischen pathologischer Diagnose und den Heilungen genauer angegeben. Das klinische Bild steht im Vordergrund. Fast immer finden wir die Angabe, daß von den Fällen, die ohne Drüsenerkrankung zur Operation kommen, ein großer Prozentsatz dauernd zu heilen ist. Es geben an:

Villars et Monniquaud ${ }^{4}$ ) 7 I Proz. ohne Drüsen gegen 2 I Proz. mit Drüsen, Halstedt ${ }^{5}$ ) 70 Proz. ohne Drüsen gegen 24 Proz. mit Drüsen, Steinthal 6 ) 69-85 Proz. ohne Drüsen gegen 26,5-32 Proz. mit Drüsen, Salomon 7 ) 85 Proz. ohne Drüsen, Hildebrand ${ }^{8}$ ) 34,6 Proz. ohne Drüsen gegen I 8,4 Proz. mit Drüsen, Gebele ${ }^{9}$ ) 99 Proz. ohne Drüsen, Sprengel10) 2o Proz. ohne Drüsen gegen 9 Proz. mit Drüsen.

Auch die Autoren, die nicht genaue Zahlen geben, betonen meistens den Vorteil der Fälle ohne Drüsencarcinomentwicklung,

I) Halstedt. l. c.

2) Warren. Annals of surgery 1904, Bd. $4^{\circ}$.

3) Salomon. l. c.

4) Villars et Monniquaud. Revue franç. de méd. et de chir. 1907, Nr. II.

5) Halstedt. 1. c.

6) Steinthal. l. c.

7) Salomon. l. c.

8) Hildebrand. 1. c.

9) Gebele. 1. c.

เo) Sprengel. l. c. 
der bei den fortgeschritteneren sich auch durch die radikalste Operation nicht einholen läßt.

Ich möchte nunmehr am Material unserer Klinik der Frage nachgehen, ob durch die radikalere Operation überhaupt ein Fortschritt erzielt worden ist. Es gibt über das Rostocker Material bereits zwei Arbeiten, von Stephan und von Schröder ${ }^{1}$ ), die die Fälle von I 875-r 900 behandeln; es sind im ganzen 347. Bei den 50 ersten Fällen, die Stephan ${ }^{2}$ ) aus der Trendelenburgschen Zeit veröffentlicht, wurde prinzipiell nur die Brust amputiert, die Achselhöhle wurde nur dann ausgeräumt, wenn Drüsen zu fühlen waren. Er hat 8 Dauerheilungen, d. h. i6 Proz., darunter sind 5 ohne Achselausräumung. (Wieder ein Beweis für die üble prognostische Bedeutung der Achseldrüsen.) Madelung hat dann neben der Ausräumung der Achselhöhle prinzipiell noch die Fascie des Pectoralis maior mitgenommen, Garrè hat die oberste Schicht dieses Muskels entfernt. Schröder ${ }^{1}$ ) kommt mit seiner Gesamtstatistik auf 2I,5 dreijährige Heilungen. Es liegt nahe, den Fortschritt von I I Proz. auf das Konto der prinzipiell ausgeführten, ausgedehnten Operation zu setzen. Die meisten Fälle der Schröderschen Statistik fallen in die Zeit der Antiseptik; die Mortalität war nur in den ersten 50 Fällen größer als bei den meinigen, nämlich ıo Proz. Später war sie nur 4,6 Proz. Die Ursache der besseren Erfolge ist also mit größter Wahrscheinlichkeit in der besseren Technik zu suchen. Da der Vergleich am selben Material vorgenommen werden kann, gewinnt er bedeutend an Beweiskraft.

Ich möchte hier noch auf einen Einwurf eingehen, der gegen das Rottersche Operationsverfahren erhoben wurde, daß es nämlich die Brauchbarkeit des Arms bedeutend herabmindere. Ich habe in meine Fragebogen diesen Punkt mit aufgenommen und in fast allen Fällen gute Auskunft bekommen. Die Patientinnen, die ihren Arm nicht gebrauchen konnten, hatten zumeist Ödem des Arms, das den Lymphabfluß erschwerte und so das Gewicht des Arms vermehrte. Von 60 nach 3 Jahren Gesunden hatten nur 4 Störungen in der Beweglichkeit des betreffenden Arms.

I) Schröder. Beitr. z. klin. Chir, Bd. 45.

2) Stephan. Dissert. Rostock I88r. 
Ob uns die Rottersche Operation einen großen Fortschritt in bezug auf die Lokalrezidive gebracht hat, kann ich leider nicht feststellen; denn Schröder ${ }^{1}$ ) macht darüber keine genauen Angaben, und auch unser Material ist lückenhaft. Ob die Rezidive in der Narbe saßen, ob daneben, ist bei den Antworten der Kollegen meist nicht angegeben. Wenn man nur die Fälle mit genauen Angaben berücksichtigt, so ist das Rezidiv meist in der Narbe aufgetreten. Im ganzen sind 85 unserer Operierten an Rezidiven erkrankt, das sind 45 Proz. I7 Kranke hatten gleichzeitig eine Metastase. Die Erfolge Halsted ts${ }^{2}$ ), der nur I I Proz. Lokalrezidive hatte, und R otte $\mathrm{rs}^{3}$ ) haben wir also nicht erreicht. Metastasen sind bei 43 Personen berichtet, von denen 6 mehrere hatten. Ihrem Sitz nach verteilen sich die Metastasen folgendermaßen :

$\begin{array}{lr}\text { Allgemeine Carcinomatose : } 9 \\ \text { Pleura und Lunge: } & \text { I2 } \\ \text { Wirbelsäule: } & 4 \\ \text { Femur: } & 4 \\ \text { Gehirn: } & 2 \\ \text { Leber : } & 8 \\ \text { Magen : } & 4 \\ \text { Darm: } & 2 \\ \text { Peritoneum : } & \text { I } \\ \text { Andere Mamma : } & 3 \\ \text { Andere Achselhöhle: } & 3\end{array}$

Rezidivoperationen wurden bei uns bei 52 Patientinnen ausgeführt. Dabei war 3 mal auch die zweite Mamma und $3 \mathrm{mal}$ auch die andere Achselhöhle ergriffen. Von diesen Kranken sind 3 nach der Rezidivoperation über 5 Jahre gesund geblieben. Ein Fall verdient besondere Beachtung.

Vor 12 Jahren erkrankte Frau L. an einem linkseitigen Mammacarcinom, das mit Fortnahme des Pectoralis major operiert wurde; im folgenden und nächstfolgenden Jahre traten Lokalrezidive auf, die operiert wurden. Im 4. Jahr zeigte sich ein Carcinom in der rechten Mamma, die ebenfalls radikal operiert wurde. Patientin blieb nun über

r) Schröder. I. c.

2) $\mathrm{H}$ a lstedt. l. c.

3) Rotter. 1. c. 
Zur Statistik der operativen Dauerheilungen des Mammacarcinoms. I79

7 Jahre rezidivfrei. Im Sommer I9I I trat ein Gibbus in der 'Lendenwirbelsäule auf, mit welchem bis April I9ı 3 nur neuralgische Schmerzen in den Beinen verbunden waren; dann trat plötzlich Lähmung beider Beine ein. Frau L. ist noch am Leben (März 19I4).

Zum Schluß möchte ich noch einmal unsere Erfahrungen kurz zusammenfassen:

I. Läßt sich auch nicht mit absoluter Sicherheit von der Dauerheilung eines Mammacarcinoms sprechen, so gehören doch die Erkrankungen nach Ablauf des 5. Jahres zu den Seltenheiten. Eine große Wahrscheinlichkeit der Dauerheilung besteht schon nach 3 Jahren. Von unseren Kranken lebten rezidivfrei nach 3 Jahren 32,7 Proz., nach 5 Jahren 28 Proz. 4 Spätmetastasen traten nach Ablauf der 5 Jahre noch auf, d.h. bei 9,4 Proz. der „Geheilten“.

2. Die Fälle ohne Achseldrüsenerkrankung geben die weitaus beste Prognose (68,5 Proz. Dauerheilungen). Sie setzen sich zusammen a) aus den früh entdeckten Tumoren aller Formen des Carcinoms, b) aus den Tumoren mit langsamem Wachstum. Unter diesen scheinen die Adenocarcinome an erster Stelle zu stehen.

3. Die Operationsmethode von R ot ter hat mit großer Wahrscheinlichkeit zur Hebung der Operationsresultate beigetragen.

4. Jeder Fall ist so früh wie möglich zur Operation zu bringen.

Ich lasse hier zum Vergleich die Resultate anderer Autoren folgen, die auf ein Triennium der Rezidivfreiheit abgestimmt sind; wo eine andere Zeit gewählt ist, steht sie unter „Bemerkungen“ daneben. Bei den mit $\left(^{*}\right)$ bezeichneten Namen habe ich nicht die vom Autor angegebene Zahl genannt, sondern sie nach dem Vorschlage von Cheyn $\mathrm{e}^{1}$ ) abgeändert. Ich halte es gleich ihm nicht für richtig, die Zahl der Heilungen in ein Verhältnis zur Zahl sämtlicher Operierten zu setzen; nur diejenigen können benutzt werden, bei denen seit der Operation schon drei Jahre verstrichen sind; dadurch wenden die Prozentsätze in einem Teil dieser Arbeiten bedeutend günstiger. Andererseits haben einige Autoren, die nur von einem Teil ihrer Operierten Auskünfte erhalten haben, bei der Aufstellung ihrer Prozentsätze die Ver-

I) Cheyne. Lancet I890, Februar. 
schollenen einfach vernachlässigt. Da es meiner Meinung nach leichter ist, über Überlebende etwas zu erfahren, als über längst Verstorbene, so wird durch das Weglassen der oben erwähnten Fälle die Statistik zu günstig. Wo dies besonders stark hervortritt, habe ich eine entsprechende Anmerkung gemacht.

Aus der Zusammenstellung ergibt sich im Durchschnitt Besserung der Resultate in den letzten 20 Jahren. Einen allgemeinen Prozentsatz der Heilbarkeit des Mammacarcinoms aufzustellen, verbietet die Verschiedenheit des Materials.

\begin{tabular}{|c|c|c|c|}
\hline & Zahl & $\%$ & Bemerkungen \\
\hline I. v. Winiwarter 1878 Zürich-Wien-Billroth) & 89 & 9,0 & j* \\
\hline 2. Henry 1879 (Breslau-Fischer) & 86 & 15,0 & $*$ \\
\hline 3. Oldekop I 879 (Kiel-Esmarch) & $17 \mathbf{I}$ & 14,0 & $*$ \\
\hline 4. Sprengel I88I (Halle-Volkmann) & I3I & I I,O & \\
\hline 5. Schmid H. I887 (Berlin, Augusta-Hospital-Küster) & 132 & 15,0 & $*$ \\
\hline 6. Rotter I 887 (Berlin-v. Bergmann) & 114 & 32,0 & $\begin{array}{c}\text { Nur } 43 \text { Falle ver- } \\
\text { wertet }\end{array}$ \\
\hline 7. Hildebrandt 1887 (Göttingen-König) & I 36 & 23,0 & $*$ \\
\hline 8. Schmidt G. B. I 889 (Heidelberg-Czerny) & 83 & 9,6 & Nach 5 Jahren $9 \%$ \\
\hline 9. Fink I 889 (Prag-Gussenbauer) & 253 & 16,7 & \\
\hline 10. Poulsen I 891 (Kopenhagener Stadthospital) & 325 & 20,0 & \\
\hline I I. Dietrich I 892 (Straßburg-L_ücke) & 95 & $\mathbf{1 6 , 0}$ & \\
\hline I 2. Horner I 884 (Zürich-Krönlein) & 144 & I 9,4 & \\
\hline I3. JoerB 1896 (Greifswald-Helferich) & 98 & 28,5 & \\
\hline 4. Meier I896 (Bern-Kocher) & 212 & 31,0 & \\
\hline 15. Jones u. Platt 1895 (Manchester royal infirmary) & $5 \xi$ & 18,0 & Über 5 Jhr. II, $5 \%$ \\
\hline I6. Cheyne W. I 896 (Kings College Hospital,London) & $2 \mathrm{I}$ & 57,0 & \\
\hline 17. Mahler 1900 (Heidelberg-Czerny) & $\mathbf{1 3 3}$ & 21,0 & \\
\hline 18. Gebele rgor (München-Angerer) & 189 & 16,9 & \\
\hline 9. Gulecke rgor (Berlin-v. Bergmann) & 382 & 28,8 & $\begin{array}{l}322 \text { Verschollene } \\
\text { vernachlässigt! }\end{array}$ \\
\hline 20. Rosenstein I9oI (Wien-v. Eiselsb & 145 & 22,7 & \\
\hline 21. Barker 1900 (Univ. College HospitaI, London) & 86 & 33,7 & Über 5 Jhr. $16,2 \%$ \\
\hline
\end{tabular}

2) Henry. Dissertat. Breslau I879. Ref. Zentralbl. f. Chir. 1882.

6) Rotter. Münchn. med. Wochenschr. $1887, \mathrm{Nr} .49$ u. 50.

9) F in k. Deutsche Zeitschr. f. Heilk. I889, Bd. 9.

13) Joerss. Deutsche Zeitschr. f. Chir. I896, Bd. 47.

14) Meier. Kochers Operationslehre.

15) J ones and Platt. Lancet 1895, August.

17) Mahler. Beitr. z. klin. Chir. I90o, Bd. 2r.

2I) B arker. Lancet 1900, September. 
Zur Statistik der operativen Dauerheilungen des Mammacarcinoms. I $8 \mathrm{I}$

\begin{tabular}{|c|c|c|c|}
\hline & Zahl & $\%$ & Bemerkungen \\
\hline 22. Schmidt P. I904 (Göttingen ect.-Braun) & I62 & 28,3 & $\begin{array}{l}53 \text { Verschollene } \\
\text { vernachlässigt }\end{array}$ \\
\hline 23. Meissl I904 (Wien) & 247 & 18,0 & \\
\hline 24. Schwarz 1905 (Graz-v. Hacker) & 186 & 14,5 & \\
\hline $\begin{array}{l}\text { 25. Schröder } 1905 \text { (Rostock-Trendelenburg, Made- } \\
\text { lung u. Garré) }\end{array}$ & 347 & 215 & \\
\hline 26. Wunderli 1906 (Spital St. Gallen) & 183 & 14,0 & \\
\hline 27. Steiner 1906 (Budapest-Dollinger) & 108 & Ió,o & \\
\hline 28. Scheu 1907 (Breslau-v. Mikulicz) & 233 & 20,2 & \\
\hline 29. Halsted 1907 (John Hopkins Hosp., Baltimore) & 232 & 38,8 & Nach $5 \mathrm{Jhr} .32,3 \%$ \\
\hline 30. Finsterer 1907 (Wien-v. Hochenegg) & 606 & 15,3 & \\
\hline 31. Greenough I907 (Massach. Gen. Hosp. Boston) & 376 & 18,0 & $\begin{array}{l}\text { 40 Verschollene } \\
\text { vernachlässigt }\end{array}$ \\
\hline 32. Ochsner 1907 (Augustana Hospital Chicago) & 164 & 31,5 & \\
\hline 33. Jacobson 1907 (Syracuse New York) & $7 \mathbf{I}$ & 49,0 & \\
\hline 34. Oliver 1907 (America, Cincinnati Ohio) & 35 & 34,3 & \\
\hline 35. Cabot I9o7 (Boston Privat-Praxis) & 42 & 33,3 & \\
\hline 36. Depage 1908 (Belgien) & $62 !$ & 48,0 & \\
\hline 37. Villars et Monniquaud igo8 (Lyon). & 50 & 54,0 & \\
\hline 38. Le Dentu 1908 (Frankreich) & 47 & 47,9 & Nach 5 Jhr. $32,2 \%$ \\
\hline 39. Heurteaux I908 (Nantes) & 284 & 43,3 & Nach 4 Jahren \\
\hline 40. Ribera y Sans rgos (Madrid) & 135 & 40,0 & \\
\hline 4I. Borelius I908 (Lund) & 77 & 35,0 & \\
\hline 42. Jonnescu igo8 (Bukarest) & 66 & 30,0 & \\
\hline 43. Dollinger 1908 (Budapest) & 40 & 6.0 & Op. n. Heidenhain \\
\hline & 72 & 24,7 & Op. n. Halsted \\
\hline 44. Warren 1909 (England) & 100 & 30,0 & \\
\hline 45. Leech Ig 10 (England) & 66 & 21,0 & \\
\hline 46. Paus I910 (Kopenhagen) & 70 & $3 I, 4$ & Über 5 Jhr. $18,8 \%$ \\
\hline
\end{tabular}

23) $\mathrm{M}$ e iss l. Wiener klin. Wochenschr. 1904.

28) S c h e u. Grenzgeb. f. Med. u. Chir. Supplementband 3.

32) O ch s ner. Annals of surgery 1907, Bd. 46.

33) J a cobson. Ebenda.

34) Oliver. Ebenda.

35) C a bot. Ebenda.

36) De page. 2. Congrès de la soc. int. de chir., Brüissel 1908.

38) Le Dentu. Ebenda.

40) Ribera. Ebenda.

4r) Borelius. Ebenda.

42) Jonnesku. Ebenda.

46) $\mathrm{Pa}$ us. Magasin f. laegevidenskab 1910. Ref. Hildebrands Jahresbericht 19 ro. 
I82 LINDENBERG, Zur Statistik der operativen Dauerheilungen usw.

\begin{tabular}{l|r|r|r}
\hline \hline & Zahl & $\%$ & Bemerkungen \\
\hline \hline 47. Steinthal I912 (Katharinen-Spit. Stuttgart) & 200 & 30,0 & \\
48. Schwarzkopf I912 (Prag Wölffler) & 269 & 20,8 & 57 Verschollene \\
(Innsbruck-Schloffer) & 66 & 27,7 & vernachlässigt \\
(Innsbrucker-Privatklinik & I4 & 42,8 & \\
Schloffer) & & & \\
49. Salomon 1913 (Berlin-Bier) & 212 & 33,5 & - \\
50. Lindenberg (Rostock, W. Müller) & I83 & 32,7 & Ubber 5 Jhr. 28,0\%
\end{tabular}

48) Schwarzkopf. Beitr. z. klin. Chir., Bd. So. 